\title{
MRI detects protective effects of DAPT treatment with modulation of microglia/macrophages at subacute and chronic stages following cerebral ischemia
}

\author{
XIAO-ZHU HAO $^{1 *}$, JIA-QI TIAN ${ }^{1 *}$, LE-KANG YIN $^{1}$, XIAO-XUE ZHANG $^{1}$, \\ CHAN-CHAN LI ${ }^{1}$, XIAO-YUAN FENG ${ }^{1}$, ZHEN-WEI YAO $^{1}$, MIN JIANG $^{2}$ and YAN-MEI YANG ${ }^{1}$ \\ ${ }^{1}$ Department of Radiology, Huashan Hospital, Fudan University, Shanghai 200040; ${ }^{2}$ Institutes of Science \\ and State Key Laboratory of Medical Neurobiology, Fudan University, Shanghai 200032, P.R. China
}

Received November 14, 2016; Accepted June 8, 2017

DOI: $10.3892 / \mathrm{mmr} .2017 .7200$

\begin{abstract}
Notch homolog 1 (Notch 1) signaling is regarded as a potential therapeutic target for modulating the inflammatory response and exhibiting neuroprotective effects in cerebral injury following stroke. $\mathrm{N}$-[N-(3,5-difluorophenacetyl)-1-alanyl]-S-phenylglycine tbutylester (DAPT) efficiently inhibits activation of the Notch 1 signaling pathway in microglia and may protect brain tissue from ischemic damage. However, the temporal proliferation and morphological alterations of microglia/macrophages throughout progression of the disease, as well as the comprehensive alterations of the whole brain following DAPT treatment, remain to be elucidated. The present study evaluated
\end{abstract}

Correspondence to: Mrs. Yan-Mei Yang, Department of Radiology, Huashan Hospital, Fudan University, 12 Middle Wulumuqi Road, Shanghai 200040, P.R. China

E-mail: yym9876@sohu.com

${ }^{*}$ Contributed equally

Abbreviations: aSVZ, anterior subventricular zone; NICD, Notch intercellular domain; bHLH, basic helix-loophelix; HES-1, enhancer of split 1; DAPT, N-[N-(3,5difluorophenacetyl)-1-alanyl]-S-phenylglycine t-butylester; MCAO, middle cerebral artery occlusion; MRI, magnetic resonance imaging; DKI, diffusion kurtosis imaging; IF, immunofluorescence; MCA, middle cerebral artery; CCA, common carotid artery; ECA, external cerebral artery; ICA, internal cerebral artery; LV, lateral ventricle; TR, Repetition Time; TE, Echo Time; FOV, Field of View; ST, slice thickness; NA, number of average; PBS, phosphate-buffered saline; $\mathrm{HLV}$, hemispheric lesion volume; HV, hemisphere volume; LV, lesion volume; ROI, regions of interest; CTX, cortex; STR, striatum; FA, fractional anisotropy; MD, mean diffusion; Da, axonal diffusion; MK, mean kurtosis; SD, standard deviation; SI, signal intensity; TNF, tumor necrosis factor

Key words: Notch, MCAO, DAPT, microglia/macrophage, striatum, magnetic resonance imaging the temporal proliferation and the morphological alterations of microglia/macrophages over the period of the subacute and chronic stages, in addition to dynamic alterations of brain tissue, using the magnetic resonance imaging (MRI) method, following DAPT treatment. Sprague-Dawley rats $(n=40)$ were subjected to $90 \mathrm{~min}$ of middle cerebral artery occlusion and were treated with DAPT $(n=20)$ or acted as controls with no treatment $(n=20)$. The two groups of rats underwent MRI scans prior to the induction of stroke symptoms and at $24 \mathrm{~h}$, 7, 14, 21 and 28 days following the stroke. A total of five rats from each group were sacrificed at 7, 14, 21 and 28 days following induction of stroke. Compared with control rats, the MRI data of the ipsilateral striatum in treated rats revealed ameliorated brain edema at the subacute stage and recovered brain tissue at the chronic stage. In addition to this, treatment attenuated the round-shape and promoted a ramified-shape of microglia/macrophages. The present study confirmed the protective effect of DAPT treatment by dynamically monitoring the cerebral alterations and indicated the possibility of DAPT treatment to alter microglial characteristics to induce a protective effect, via inhibition of the Notch signaling pathway.

\section{Introduction}

Stroke is the second leading cause of disability and mortality across the world, and only a minority of stroke patients can benefit from the thrombolytic therapy due to the narrow time window and the increased risk of hemorrhage $(1,2)$. Therefore, strategies, which focus on protecting and repairing the damaged brain after stroke, are urgently needed. Based on studies that Notch 1 signaling is vital in the regulation of regenerative responses after ischemia and reperfusion, it is regarded as a potential therapeutic target for modulating inflammatory reaction and releasing cerebral injury after stroke (3-5).

Notch 1 signaling is composed of Notch 1 receptor and Notch 1 ligands $(3,6,7)$, which was implicated schematically in Fig. 1. Notch 1 receptor is a single-pass transmembrane protein which is found on the surface of neural progenitors (3), immature neurons $(6,8)$, and glial cells (9-11) of hippocampus and anterior subventricular zone (aSVZ) $(3,12)$. When activated by the ligands expressed on neighboring cells, 
Notch 1 is cleaved by the $\gamma$-secretase complex, and releases a Notch intracellular domain (NICD) that translocates into nucleus and activates transcription factors, which eventually worsens brain damage by augmenting inflammatory reaction of microglia and stimulating of peripheral leukocytes and lymphocytes $(4,8,9,13-15)$. Based on these evidences, attenuation of the canonical Notch 1 signaling is beneficial to the ischemic cerebral tissue. There has been increasing interest on N-[N-(3,5-difluorophenacetyl)-1-alanyl]-S-phenylglycine t-butylester (DAPT), which can protect brain tissue from ischemic damage with efficiently inhibiting activation of the Notch 1 signaling in microglia (9).

It is well documented that microglia undergo progressive morphological transformation and functional alterations over different stages after stroke (16), that round-shaped microglia was detrimental to the brain tissue via release the pro-inflammatory cytokines while the ramified-shaped one was beneficial to the damaged tissue by releasing neurotrophic factors. However, the previous studies put focus on the attenuation of microglia via the inhibition of Notch signaling. So the temporal proliferation and morphological changes of microglia over stages, as well as the comprehensive outcome of the whole brain tissue following DAPT treatment, is unknown. Magnetic resonance imaging (MRI), as the non-invasive and more advanced method, can be used to longitudinally monitor the comprehensive changes of the brain tissue. In this study, for the first time, we evaluated the temporal proliferation and morphological changes of microglia/macrophage over the period of subacute stage and chronic stage and evaluated the dynamic changes of brain tissue with the MRI method following DAPT treatment.

\section{Materials and methods}

Animals. All animal procedures performed in this study were approved by Fudan University of Institutional Animal Care and Use Committee, and every effort was made to minimize suffering and to reduce the number of animals used. A total of 40 Male Sprague-Dawley rats weighing 260-270 g were divided into two groups: Control group $(n=20)$ and treated group $(n=20)$. All rats were subjected to a 90-min of (middle cerebral artery occlusion) MCAO and were performed with MRI before and at $24 \mathrm{~h}, 7,14,21$ and 28 days after stroke. At the same time, five rats of each group were decapitated at 7, 14, 21 and 28 days after stroke respectively for immunofluorescence staining (IF). Fig. 2 shows the time schedule for experimental procedures in all groups.

Stroke model. Rats were anesthetized with an intraperitoneal injection of $10 \%$ chloral hydrate under spontaneous inspiration, and the body temperature was continuously monitored at $37 \pm 0.5^{\circ} \mathrm{C}$ during the surgical procedures. For all rats, the left middle cerebral artery (MCA) was occluded for $90 \mathrm{~min}$. In details, rats were immobilized by a tooth holder and with binding of all limbs, followed by the insertion of a 4.0 silicon-coated polypropylene suture into the left internal carotid artery (ICA) through the external carotid artery (ECA) and common carotid artery (CCA) to block blood flow to the MCA. After $90 \mathrm{~min}$, the filament was withdrawn from the ICA to allow reperfusion. The follow-up T2 MRI was acquired

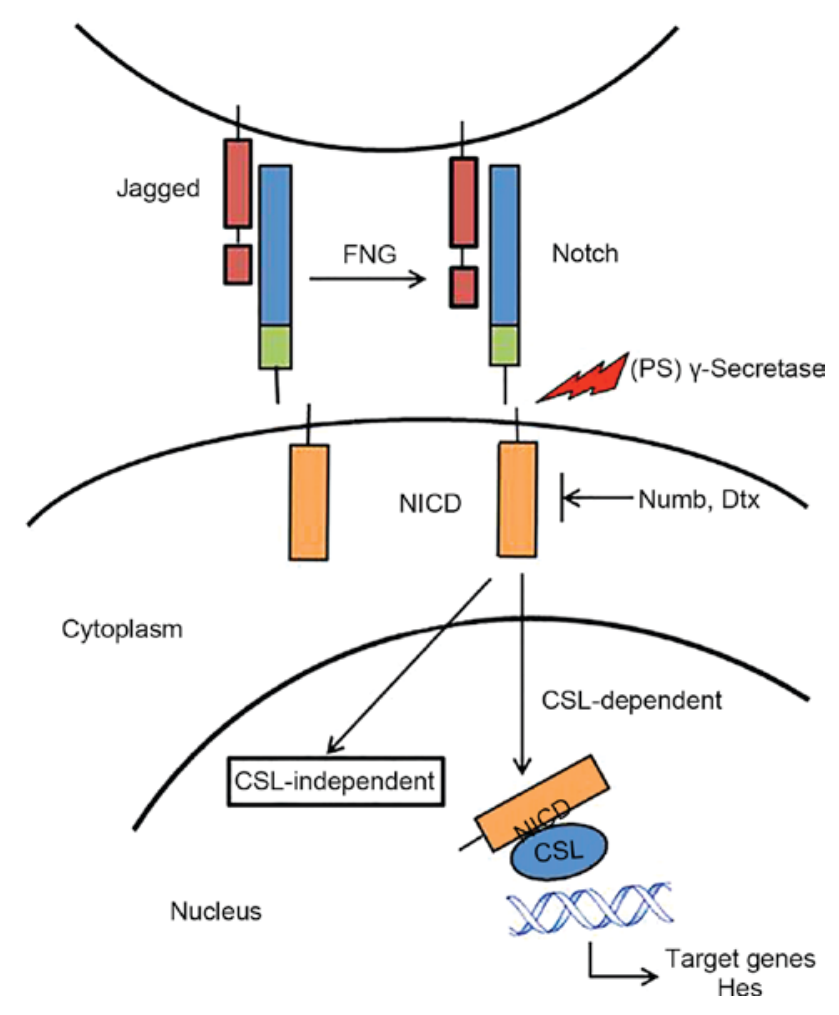

Figure 1. Schematic representation of Notch1 signaling pathway in adult rat brain.

under the anesthetized circumstances to check the occlusion of the left hemisphere $24 \mathrm{~h}$ post-stroke.

Administration of DAPT. DAPT powder (Sigma-Aldrich, St. Louis, MO, USA), was dissolved in DMSO to prepare concentrations of $8.3 \mathrm{mg} / \mathrm{ml}$ (17). DAPT solution $(0.03 \mathrm{mg} / \mathrm{kg})$ was stereotactically injected into the lateral cerebral ventricle (LV) for treated group rats at 3 days after stroke. Rats were anesthetized and placed in a stereotactic holder and immobilized by earplugs and a toothholder. A burr hole was drilled in the skull, $4.0 \mathrm{~mm}$ deep into the pial surface, $-2.0 \mathrm{~mm}$ anteroposterior relative to the bregma, and $1.0 \mathrm{~mm}$ lateral to the midline, according to Paxinos and Watson (1998) (18). With a $2.0 \mu \mathrm{l}$ Hamilton syringe, DAPT administration was finished within $3 \mathrm{~min}$ and the needle was left in place for $4 \mathrm{~min}$ to prevent leakage along the injection track. The control group rats received the same volume PBS at the same time point.

Magnetic resonance imaging (MRI). Multimodal MRI of the rat, including relaxation time imaging and diffusion imaging, was temporally conducted before and after $24 \mathrm{~h}, 7,14,21$ and 28 days of transient cerebral ischemia. Prior to MRI, animals were anesthetized by the same procedure as described for the MCAO model (see above). The body temperature was continuously monitored at $37 \pm 0.5^{\circ} \mathrm{C}$, at the same time, blood oxygen saturation and heart rate were monitored during MRI procedures. The MRI measurements were performed in a 3.0-T horizontal magnet (Discovery MR750; GE Medical Systems, Milwaukee, WI, USA) with a 60-mm-diameter gradient coil (Magtron Inc., Jiangyin, China).

T2-weighted (wt) MR images were acquired by a fast spin-echo sequence with the following parameters: Repetition 


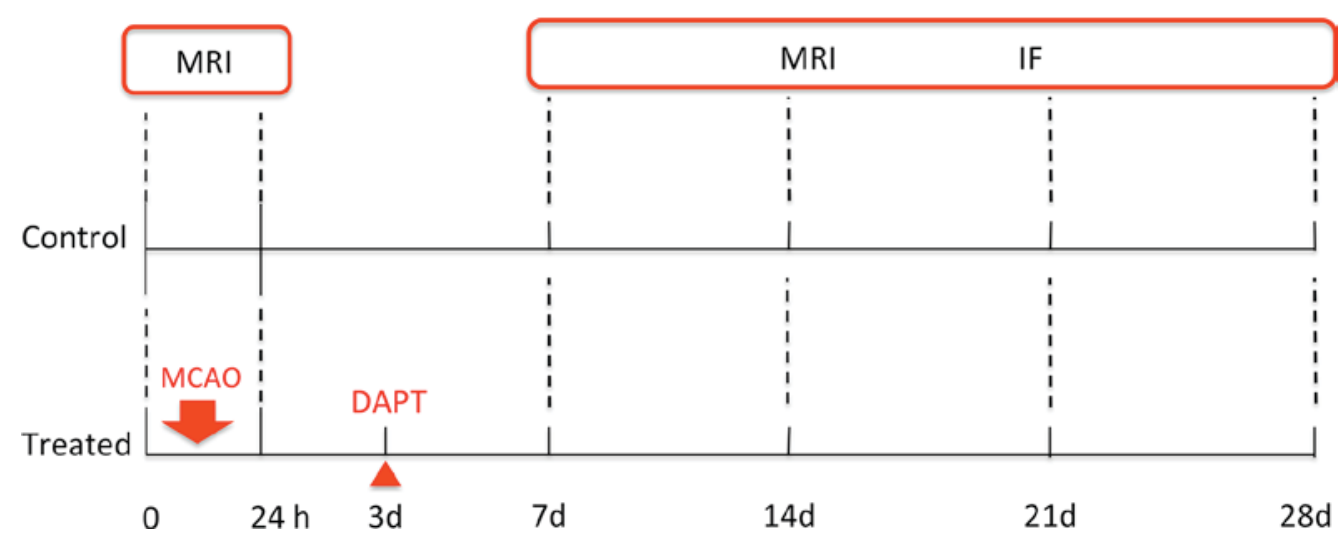

Figure 2. Experimental procedure. Forty rats were subjected to a 90-min of MCAO and divided into two groups: Control group (n=20) and treated group $(\mathrm{n}=20)$. Rats suffered from transient cerebral ischemia were performed with MRI before and at 24 h, 7, 14, 21 and 28 days after stroke. At the same time, 5 rats of each group were decapitated at 7, 14, 21 and 28 days, respectively for IF.

Time $(\mathrm{TR}) /$ Echo Time $(\mathrm{TE})=4,000 / 96 \mathrm{msec}, \mathrm{scan}$ time $=3 \mathrm{~min}$, Field of View $(\mathrm{FOV})=6 \times 6 \mathrm{~cm}$, matrix $=256 \times 256$, slice thickness $(\mathrm{ST})=1.8 \mathrm{~mm}$, spatial resolution $=0.24 \times 0.24 \times 1.8 \mathrm{~mm}^{3}$, inter-slice distance $=2 \mathrm{~mm}$, number of slices $=15$, and number of average $(\mathrm{NA})=2$.

For the DKI acquisition, diffusion weighted images were acquired with $\mathrm{TR} / \mathrm{TE}=4,000 / 84 \mathrm{msec}, \mathrm{FOV}=6 \times 6 \mathrm{~cm}$, $\mathrm{ST}=1.8 \mathrm{~mm}$, inter-slice distance $=2 \mathrm{~mm}$, matrix $=64 \times 64$, in-plane voxel size $=234 \times 234 \mu \mathrm{m}, \mathrm{NA}=4$. The number of gradient directions was 15 with the duration of each of the diffusion gradients $(\delta)$ being $4 \mathrm{~ms}$ with a temporal spacing of $23 \mathrm{msec}(\Delta)$ between the two diffusion gradients. Two b-values $\left(1,000\right.$ and $\left.2,000 \mathrm{sec} / \mathrm{mm}^{2}\right)$ were acquired for each direction following the acquisition of five images acquired at $\mathrm{b}=0 \mathrm{sec} / \mathrm{mm}^{2}$.

Immunostaining. Removed brain samples of rats were post-fixed in $4 \%$ paraformaldehyde for $24 \mathrm{~h}$, and then were vitrified in 20 and $30 \%$ sucrose solutions for $24 \mathrm{~h}$ and 3 days, respectively. Coronal brain sections of all rats were obtained using a cryostat (RM2135; Leica, Mannheim, Germany). Immunofluorescence was performed on cryosections $(20 \mu \mathrm{m})$ : Approximately 1.70 to $-4.80 \mathrm{~mm}$ to Bregma according to Paxinos and Watson (18). In details, sections were washed three times with phosphate-buffered saline (PBS, $\mathrm{pH}=7.4$ ). Sections were then blocked from non-specific binding with $10 \%$ normal donkey serum in PBS containing $0.3 \%$ Trition-X-100 (Sigma-Aldrich, St. Louis, MO, USA) for $2 \mathrm{~h}$ at room temperature. After aspiration of block solution, sections were incubated with primary antibodies of rabbit polyclonal anti-NICD (1:500; Abcam, Cambridge, MA, USA) and mouse monoclonal anti-CD11b (1:200; AbD Serotec, Kidlington, UK). Primary antibodies were incubated overnight with sections at $4^{\circ} \mathrm{C}$. Balaced at room temperature for about $30 \mathrm{~min}$, sections were rinsed with PBS for 5 min with 3 times. Sections were then incubated with secondary antibodies as Alexa Fluor 488and 568- conjugated donkey anti-mouse or anti-rabbit (1:200; Life Technologies, Carlsbad, CA, USA). Rinsed with PBS for 5 min with 3 times, sections were then counterstained with DAPI (1:1,000; Sigma-Aldrich, St. Louis, MO, USA). At last, all the sections were rinsed with PBS for 5 min with 3 times, and coverslipped with mounting medium. Sections from two groups were processed together in the same batches to minimize staining variability.

\section{Image processing and quantitative analysis}

MRI. The lesion volume was represented as edema-corrected hemispheric lesion volume $\left(\% \mathrm{HLV}^{\mathrm{e}}\right)$, which was firstly described by Gerriets with ADC map and $\mathrm{T}_{2}$-wt $\mathrm{MR}$ images (19). The lesion volume was calculated on T2 MR images as follows: $\% \mathrm{HLV}^{\mathrm{e}}=\left(\mathrm{HV}_{\mathrm{c}}-\left(\mathrm{HV}_{\mathrm{i}}-\mathrm{LV}^{\mathrm{u}}\right) / \mathrm{HV}_{\mathrm{c}} \mathrm{x} 100 \%\right.$, where $\mathrm{HV}_{\mathrm{c}}$ and $\mathrm{HV}_{\mathrm{i}}$ indicate volume of the contralateral and ipsilateral hemisphere respectively, and $\mathrm{LV}^{\mathrm{u}}$ indicate the uncorrected lesion volume (Fig. 3A). LV ${ }^{\mathrm{u}}$, was determined from a suitable number of noncontiguous coronal T2 MR images covering the entire ischemic area, by manual tracing of the hyperintense lesions. $\mathrm{HV}_{\mathrm{c}}$ and $\mathrm{HV}_{\mathrm{i}}$ were determined with anatomical landmarks from the same images. The areas were then summed and multiplied by slice thickness. Images of animals were excluded from further analysis with no cortical $\mathrm{T} 2$ lesions or very large hyperintense lesions extending to the contralateral hemisphere.

Then regions of interest (ROIs) including cortex (CTX) and striatum (STR) were selected for analysis of T2 signal intensity (SI) and DKI parameters, as gray matter and white matter may have different pathological evolution after ischemia. For T2 SI analysis of CTX, the ROI was placed on ipsilateral cortex with four noncontiguous T2 MR images mostly covering the ischemic lesion. And for striatum analysis, the ROI was placed on ipsilateral striatum with only one T2 MR image, which can cover majority of the lesion and avoid effects of adjacent regions.

To assess the DKI parameters, axial T2 MR images were used for anatomical references to assess the topography of infarction. All parameters were calculated from same images as for T2 SI analysis of CTX and STR respectively, in which ROIs encompass the ischemic lesion with pixel values distinctly higher than contralateral hemisphere. Contralateral ROI regions were drawn on the contralateral hemisphere according to the size and shape of the ischemic ROIs. For all images, FA, MD, $\mathrm{D}_{\mathrm{a}}$ and $\mathrm{MK}$ values were measured. Entire ROI analysis was repeated twice to ensure reproducibility of the results. Percent changes of T2 SI and DKI parameter values of both CTX and STR were computed as: $100 x\left(\mathrm{X}_{\mathrm{i}}-\mathrm{X}_{\mathrm{c}}\right) / \mathrm{X}_{\mathrm{c}}$, where $\mathrm{X}$ represents an averaged metric. 
A
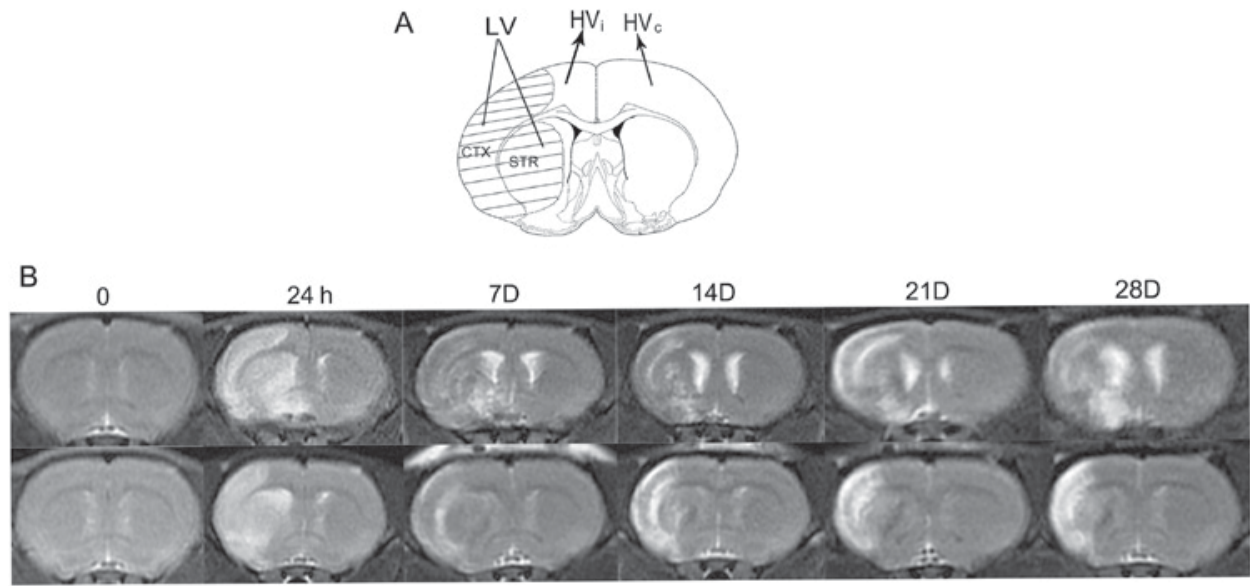

C

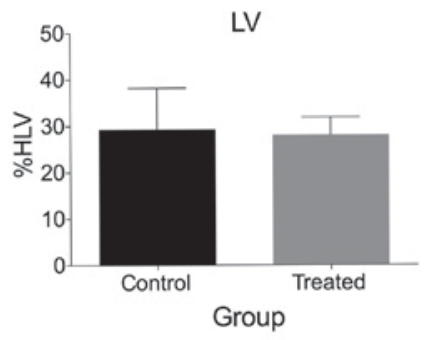

D



Figure 3. Longitudinal observation of T2 MR images for rats before and after MCAO with and without DAPT treatment. (A) Anatomical reference showing the ROI. (B) The evolution changes in T2 MR images of two group rats. (C) The lesion volume of rats with and without DAPT treatment at $24 \mathrm{~h}$ after MCAO. (D) The temporal profiles of percent changes of T2 SI for CTX and STR of two group rats. ${ }^{*} \mathrm{P}<0.05 ;{ }^{* *} \mathrm{P}<0.01$.

Immunostaining. For histological images, sections were scanned with the 10x objective using fluorescence microscope (Olympus PX51; Olympus Corporation, Shinjuku-ku, Japan). With Image J (National Institutes of Health, Bethesda, MD, USA), the acquired images were assessed semi-quantitatively by measuring optical density of positively stained cells of the selected ROI, which was selected at the lesion core of both cortex and striatum. The ipsilateral versus contralateral differences in gray levels were converted to a percentage of the contralateral value.

Statistical analysis. Unpaired t-tests were performed for two groups comparison at different time points. In statistical tests, differences were considered significant when $\mathrm{P}<0.05$ and data were presented as mean \pm SD. Statistical analysis was performed using Prism, version 6.0 (GraphPad Software Incorporated, La Jolla, CA, USA).

\section{Results}

T2-wt MR imaging. The spatiotemporal dynamics of $\mathrm{T} 2 \mathrm{~W}$ images of two group rats showed that the ischemic lesion was formed and presented hyperintensity after ischemia (Fig. 3B). And the lesion volume showed a good consistency between the control group and the treated group at $24 \mathrm{~h}$ following ischemia (Fig. 3C).
At the same time, the temporal evolution of the percent change of T2 SI for STR and CTX after reperfusion showed comparisons of cerebral tissue changes between two group rats at different time points (Fig. 3D). There was no statistical difference of the percent change of T2 SI for both STR and CTX between two group rats before and after $24 \mathrm{~h}$ following reperfusion. For STR, at 7 days after reperfusion, the percent change of T2 SI was significantly lower in the treated group than that in the control group $(\mathrm{P}<0.05)$. Then at 14 days after stroke, though the cystic region occurred to STR in both two group rats and the percent change of T2 SI showed no statistical difference, it further enlarged in rats without DAPT treatment while disappeared in rats with DAPT treatment at later time points. Simultaneously, the difference of percent change of T2 SI was enlarged that it was significantly elevated in control rats while gradually recovered to the baseline in treated rats at 21 days $(\mathrm{P}<0.01)$ and 28 days $(\mathrm{P}<0.01)$. However, for CTX, the cystic region was formed in both control rats and treated rats from 7 days to 28 days, and there was no statistical difference of the percent change of T2 SI between two groups.

Diffusional kurtosis imaging. Based on the results detected by T2 MR imaging, we next measured the microstructural differences between rats with or without DAPT treatment 




Figure 4. DKI maps of rats before and after MCAO with and without DAPT treatment. (A) The evolution changes in MD maps of two group rats. (B) The evolution changes in Da maps of two group rats. (C) The evolution changes in FA maps of two group rats. (D) The evolution changes in MK maps of two group rats.
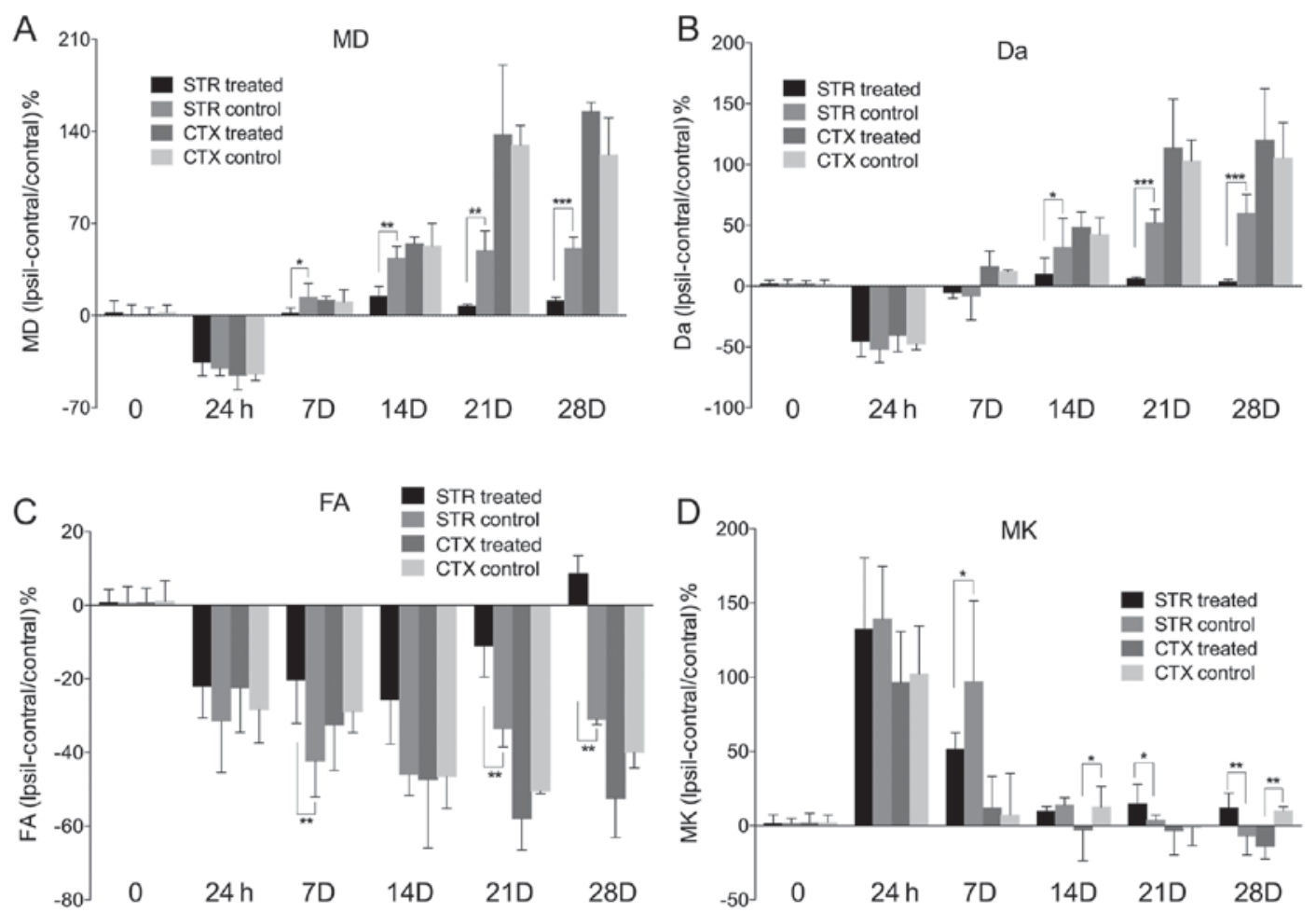

Figure 5. Quantitative characterization of DKI parameters of rats before and after MCAO with and without DAPT treatment. (A) The temporal profiles of percent changes of MD for CTX and STR of two group rats. (B) The temporal profiles of percent changes of Da for CTX and STR of two group rats. (C) The temporal profiles of percent changes of FA for CTX and STR of two group rats. (D) The temporal profiles of percent changes of MK for CTX and STR of two group rats. ${ }^{*} \mathrm{P}<0.05 ;{ }^{* *} \mathrm{P}<0.01 ;{ }^{* * *} \mathrm{P}<0.001$.

(Fig. 4), combining with statistical analysis of diffusion parameters, which showed no difference between two groups on all DKI parameters before and at $24 \mathrm{~h}$ after stroke (Fig. 5).
$\mathrm{MD}$ and Da were decreased in all rats at $24 \mathrm{~h}$ after reperfusion. They reversely and gradually increased at 7, 14, 21 and 28 days in rats without DAPT treatment, while recovered to 


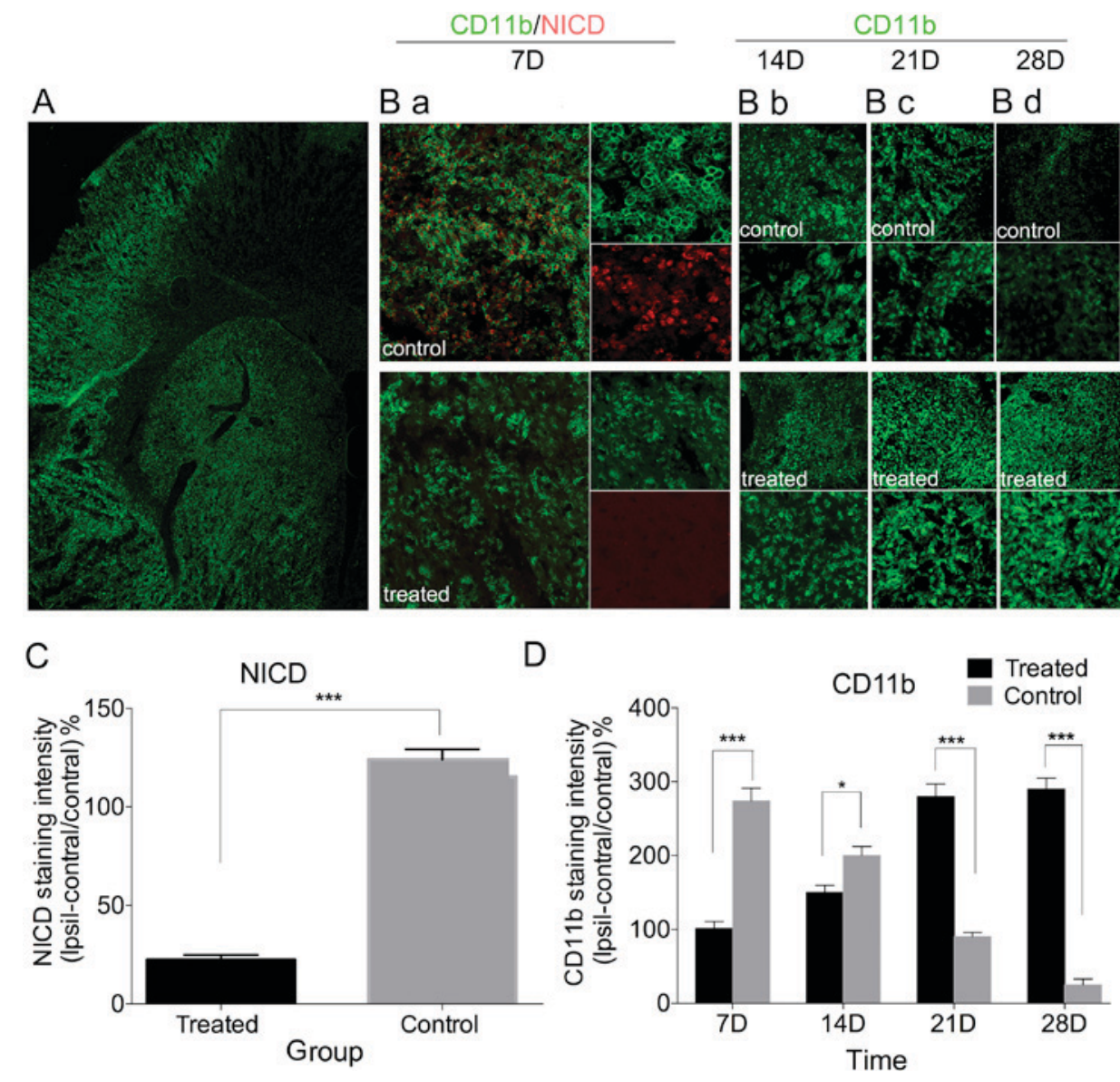

Figure 6. Microglia/macrophage and Notch 1 activity in rats after MCAO with and without DAPT treatment. (A) The 'photomerged' image shows the location of microglia/macrophage. (Ba) Immunostaining of CD11b and NICD in rats at 7 days after MCAO with and without DAPT treatment. (Bb) Immunostaining of $\mathrm{CD} 11 \mathrm{~b}$ in rats at 14 days after MCAO with and without DAPT treatment. (Bc Immunostaining of CD11b in rats at 21 days after MCAO with and without DAPT treatment. (Bd) Immunostaining of CD11b in rats at 28 days after MCAO with and without DAPT treatment. (C) Quantification of NICD fluorescence intensity in STR of rats at 7 days after MCAO with and without DAPT treatment. (D) Quantification of CD11b fluorescence intensity in STR of rats after MCAO with and without DAPT treatment. ${ }^{*} \mathrm{P}<0.05 ;{ }^{* * *} \mathrm{P}<0.001$.

the baseline in rats with DAPT treatment (Fig. 4A and B), and the analysis showed statistical differences between two groups (7D, $\mathrm{P}<0.05 ; 14 \mathrm{D}, \mathrm{P}<0.01 ; 21 \mathrm{D}, \mathrm{P}<0.01 ; 28 \mathrm{D}, \mathrm{P}<0.001$ ) (Fig. 5A and B).

FA, in all the stroke models, was significantly decreased at $24 \mathrm{~h}$ following ischemia. Then it further reduced at 7 and 14 days, and steadied at 21 and 28 days when cystic region was formed in control rats, while gradually recovered to the baseline in treated rats from 7 to 28 days (Fig. 4C). And there were statistical differences between two group rats at 7,21 and 28 days after reperfusion $(7 \mathrm{D}, \mathrm{P}<0.01 ; 21 \mathrm{D}, \mathrm{P}<0.01 ; 28 \mathrm{D}$, $\mathrm{P}<0.001$ ) (Fig. 5C).

MK decreased in all rats at 7 days compared to $24 \mathrm{~h}$ following ischemia, which was more recovered in treated rats. It was then gradually returned to the baseline in treated rats, and being reversely lower than the baseline in control rats, while still be steadied in treated rats when cystic lesion occurred at 21 and 28 days (Fig. 4D), which showed statistical differences between two groups (21D, $\mathrm{P}<0.05 ; 28 \mathrm{D}, \mathrm{P}<0.01)$ (Fig. 5D).

For ipsilateral CTX, there was no statistical difference between rats with or without DAPT treatment at 7, 14 and 21 days after stroke. However, at 28 days after reperfusion, FA, $\mathrm{MD}$ and $\mathrm{MK}$ in treated rats showed more deviation from the baseline, which may account for atrophy of ipsilateral cortex covered by cerebrospinal fluid $(\mathrm{P}<0.05)$ (Figs. 4A, C and D, and 5A, C and D).

Notch signaling and Microglia/macrophage activation. To determine whether Notch 1 receptor of microglia/macrophage was mainly activated by brain ischemia, and to observe whether DAPT can influence the activation of microglia/macrophage, we performed the CD11b/NICD staining. Microglia/Macrophage was mainly located in the lesion area (Fig. 6A). NICD was detected in the CD11b-labeled cells in the ipsilateral CTX and STR at 7 days after reperfusion in rats with or without DAPT treatment (Fig. 6B1). And for STR, the percent change of IF intensity in both NICD and CD11b was significantly lower in treated rats than in control rats (both NICD and CD11b, $\mathrm{P}<0.001$ ) (Fig. 6C and D). More importantly, the shape of microglia/macrophage was round in control rats while be with processes in treated rats, which might be M1 phenotype and M2 phenotype of microglia/macrophage, respectively. However, histology showed that microglia/macrophage in treated rats was reversely more activated in the treated group at 14 days $(\mathrm{P}<0.05), 21 \mathrm{D}(\mathrm{P}<0.001)$ and 28 days $(\mathrm{P}<0.001)$, which was ramified with processes (Fig. 6B2, B3, B4 and D). 
Correspondence between MRI and histological measures. By MRI, we found that the recovered brain tissue, after DAPT treatment, was prominently located in the STR. At 7 days, we know that the decreased T2 SI within the ipsilateral STR was mainly accounted for cell proliferation of inflammatory cells or ameliorated brain edema. And the percent change of T2 SI was more reduced in rats receiving DAPT treatment, which might be accounted for the more proliferative cells or more ameliorated brain edema. By analysis of attenuated CD11b (+) cells within the STR in treated rats, we got the result that it was mainly ameliorated brain edema contributing to the reduced T2 SI and relative recovered diffusion parameters. Then at 14 days after reperfusion, the cystic region occurred to both two groups, and further enlarged in control rats while disappeared in treated rats at 21 and 28 days. Diffusion parameters were gradually recovered to the baseline in control rats while showed further enlargement from the normal level in treated rats. And in treated rats, histology showed that intensity of CD11b (+) cells was reversely higher than that in control rats, which suggested that the recovered brain tissue was mainly filled with M2 phenotype of microglia/macrophage.

\section{Discussion}

MR images in our study showed that the recovered tissue was mainly located at the ipsilateral STR, and there was no cyst in ipsilateral STR of two group rats at 7 days, but percent changes of T2 SI, MD, FA, MK of STR in treated rats were closer to the baseline, indicating the attenuated brain edema at the subscute stage in the ipsilateral STR of rats following DAPT treatment. Then at 14 days after reperfusion, although scattered cystic development within ipsilateral STR occurred in both control rats and treated rats, the percent changes of MD, Da and MK showed more recovery in treated rats than in control rats. The cystic area disappeared in treated rats at 21 and 28 days while further enlarged in control rats at the same period. In correspondence, percent changes of all MR parameters of the same ROI showed relative recovery in the treated group and further enlargement in the control group respectively, indicating the amelioration and recovery of the damaged brain tissue with DAPT treatment. Meanwhile, the IF result in our study showed that NICD was detected in the CD11b (+) cells in the ipsilateral STR at 7 days after reperfusion, and there was less expression of NICD in treated rats than in control rats, indicating that the Notch signaling pathway in microglia/macrophage was inhibited by DAPT. And compared with control rats, the percent change of IF intensity of CD11b in treated rats was lower at 7 days and reversely higher at 14,21 and 28 days after reperfusion. And the microglia/macrophage presented as round shape at the subacute stage and ramified shape at the chronic stage respectively.

By dynamically monitoring the cerebral changes, we confirmed that inhibition of Notch signaling in the subacute phase could promote brain recovery, which was in agreement with the previous study (3). More importantly, we further indicated the possibility of DAPT treatment to change microglia's characteristic from possible damaging effect to protective effect via inhibition of the Notch pathway, which should be a potential therapeutic goal.
Activated microglia perform dual actions after stroke that both beneficial and detrimental phenotypes coexist along the pathological environment. Different stimuli act on different microglial receptors and determine the microglial phenotype $(20,21)$. And microglia/macrophage activation is not an 'all or none' process, but rather a continuum from classical activation phenotype (M1) to alternative activation phenotype (M2), which depends on inputs and feedback signals arising from the microenvironment $(16,22)$. The early M1 phenotype of microglia/macrophage, regarded as round morphology, produces inflammation-related cytokines IL-1 and tumor necrosis factor (TNF), which are considered to be the major culprits of a robust inflammatory response leading to necrosis and axonal breakdown $(23,24)$. In our study, the round-shaped microglia/macrophage in the ipsilateral STR was attenuated at 7 days in rats following DAPT treatment, and MRI data confirmed that there was less brain edema in the ipsilateral STR of treated rats. Be different from the early M1 phenotype, the later M2 polarized microglia state, regarded as ramified morphology, releases high levels of anti-inflammatory cytokines and neurotrophic factors leading to inhibition of pro-inflammatory responses, neuroprotection and wound healing (9,23-25). In our study, the ramified-shaped microglia/macrophage was detected in the ipsilateral STR in both two group rats at chronic time points of 14, 21 and 28 days after reperfusion, and the IF intensity of CD11b was reversely higher in treated rats than that in control rats. And the MR images showed that rats in the treated group experienced decreased necrosis in the ipsilateral STR at these chronic time points. Also possibly, there were more surviving or sprouting axons inside the lesion with the increased FA and reduced Da in treated rats, representing the myelinated increased axons $(26,27)$, which needs further studies.

Finally, we confirmed the protective effects of DAPT treatment by dynamically monitoring the cerebral tissue with the in vivo MRI method, and indicated the possible transformation of microglia/macrophage phenotypes by the post-mortem IF examination. And the limits in this study, regarding the optimal therapeutic time window as well as the activity of neurogenesis and neuronal differentiation, would be our focus in the further investigations.

\section{Acknowledgements}

This study was supported by the grant of the National Natural Science Foundation of China (no. 81371521) and the Shanghai Natural Science Foundation of China (no. 09ZR1405100).

\section{References}

1. Sun F, Mao X, Xie L, Ding M, Shao B and Jin K: Notch1 signaling modulates neuronal progenitor activity in the subventricular zone in response to aging and focal ischemia. Aging Cell 12: 978-987, 2013.

2. Go AS, Mozaffarian D, Roger VL, Benjamin EJ, Berry JD, Borden WB, Bravata DM, Dai S, Ford ES, Fox CS, et al: Heart disease and stroke statistics-2013 update: A report from the American Heart Association. Circulation 127: e6-e245, 2013.

3. Oya S, Yoshikawa G, Takai K, Tanaka JI, Higashiyama S, Saito N, Kirino T and Kawahara N: Attenuation of Notch signaling promotes the differentiation of neural progenitors into neurons in the hippocampal CA1 region after ischemic injury. Neuroscience 158: 683-692, 2009. 
4. Arumugam TV, Chan SL, Jo DG, Yilmaz G, Tang SC, Cheng A, Gleichmann M, Okun E, Dixit VD, Chigurupati S, et al: Gamma secretase-mediated Notch signaling worsens brain damage and functional outcome in ischemic stroke. Nat Med 12: 621-623, 2006.

5. Zhao Y, Chen X, Ma L, Zuo Z, Zhu Z, Zhu X, Wang Q, He E, Xiong L, Pei J, et al: Electroacupuncture pretreatment induces tolerance against focal cerebral ischemia through activation of canonical Notch pathway. BMC Neurosci 13: 111, 2012.

6. Wang X, Mao X, Xie L, Greenberg DA and Jin K: Involvement of Notch1 signaling in neurogenesis in the subventricular zone of normal and ischemic rat brain in vivo. J Cereb Blood Flow Metab 29: 1644-1654, 2009.

7. de la Pompa JL, Wakeham A, Correia KM, Samper E, Brown S, Aguilera RJ, Nakano T, Honjo T, Mak TW, Rossant J and Conlon RA: Conservation of the Notch signalling pathway in mammalian neurogenesis. Development 124: 1139-1148, 1997.

8. Redmond L, Oh SR, Hicks C, Weinmaster G and Ghosh A Nuclear Notch1 signaling and the regulation of dendritic development. Nat Neurosc 3: 30-40, 2000.

9. Wei Z, Chigurupati S, Arumugam TV, Jo DG, Li H and Chan SL: Notch activation enhances the microglia-mediated inflammatory response associated with focal cerebral ischemia. Stroke 42 : 2589-2594, 2011

10. Zhang Y, He K, Wang F, Li X and Liu D: Notch-1 signaling regulates astrocytic proliferation and activation after hypoxia exposure. Neurosci Lett 603: 12-18, 2015.

11. Shimada IS, Borders A, Aronshtam A and Spees JL: Proliferating reactive astrocytes are regulated by Notch-1 in the peri-infarct area after stroke. Stroke 42: 3231-3237, 2011.

12. Nyfeler Y, Kirch RD, Mantei N, Leone DP, Radtke F, Suter U and Taylor V: Jagged1 signals in the postnatal subventricular zone are required for neural stem cell self-renewal. EMBO J 24: 3504-3515, 2005

13. Ohtsuka T, Ishibashi M, Gradwohl G, Nakanishi S, Guillemot F and Kageyama R: Hes1 and Hes5 as notch effectors in mammalian neuronal differentiation. EMBO J 18: 2196-2207, 1999.

14. Shin HM, Minter LM, Cho OH, Gottipati S, Fauq AH, Golde TE, Sonenshein GE and Osborne BA: Notch1 augments NF- $x$ B activity by facilitating its nuclear retention. EMBO J 25: 129-138, 2006.

15. Eagar TN, Tang Q, Wolfe M, He Y, Pear WS and Bluestone JA: Notch 1 signaling regulates peripheral $\mathrm{T}$ cell activation. Immunity 20: 407-415, 2004

16. Town T,Nikolic V and Tan J: The microglial 'activation' continuum: From innate to adaptive responses. J Neuroinflammation 2: 24, 2005 .
17. Li S, Zyang X, Wang Y, Ji H, Du Y and Liu H: DAPT protects brain against cerebral ischemia by down-regulating the expression of Notch 1 and nuclear factor kappaB in rats. Neurol Sci 33: $1257-1264,2012$

18. Paxinos $G$ and Watson C: The Rat Brain in Stereotaxic Coordinates. 4th edition. Academic Press, San Diego, CA, 1998.

19. Gerriets T, Stolz E, Walberer M, Müller C, Kluge A, Bachmann A, Fisher M, Kaps M and Bachmann G: Noninvasive quantification of brain edema and the space-occupying effect in rat stroke models using magnetic resonance imaging. Stroke 35: 566-571, 2004.

20. Butovsky O, Talpalar AE, Ben-Yaakov K and Schwartz M: Activation of microglia by aggregated beta-amyloid or lipopolysaccharide impairs MHC-II expression and renders them cytotoxic whereas IFN-gamma and IL-4 render them protective. Mol Cell Neurosci 29: 381-393, 2005.

21. Schwartz M, Butovsky O, Brück W and Hanisch UK: Microglial phenotype: Is the commitment reversible? Trends Neurosci 29: 68-74, 2006.

22. Giunti D, Parodi B, Cordano C, Uccelli A and Kerlero de Rosbo N: Can we switch microglia's phenotype to foster neuroprotection? Focus on multiple sclerosis. Immunology 141: 328-339, 2014

23. Gomes-Leal W: Microglial physiopathology: How to explain the dual role of microglia after acute neural disorders? Brain Behav 2: 345-356, 2012.

24. Takeuchi H, Mizuno T, Zhang G, Wang J, Kawanokuchi J, Kuno R and Suzumura A: Neuritic beading induced by activated microglia is an early feature of neuronal dysfunction toward neuronal death by inhibition of mitochondrial respiration and axonal transport. J Biol Chem 280: 10444-10454, 2005.

25. Yuan Y, Rangarajan P, Kan EM, Wu Y, Wu C and Ling EA: Scutellarin regulates the Notch pathway and affects the migration and morphological transformation of activated microglia in experimentally induced cerebral ischemia in rats and in activated BV-2 microglia. J Neuroinflammation 12: 11, 2015.

26. Jiang Q, Zhang ZG, Ding GL, Silver B, Zhang L, Meng H, Lu M, Pourabdillah-Nejed-D S, Wang L, Savant-Bhonsale S, et al: MRI detects white matter reorganization after neural progenitor cell treatment of stroke. Neuroimage 32: 1080-1089, 2006.

27. Tuor UI, Morgunov M, Sule M, Qiao M, Clark D, Rushforth D, Foniok T and Kirton A: Cellular correlates of longitudinal diffusion tensor imaging of axonal degeneration following hypoxic-ischemic cerebral infarction in neonatal rats. Neuroimage Clin 6: 32-42, 2014. 